\title{
Photoelectric recording of pigeon-peck responses to computer-driven visual displays
}

\author{
DONALD S. BLOUGH \\ Brown University, Providence, Rhode Island 02906
}

\begin{abstract}
A small photocell mounted on a pigeon's beak signalled the location of pecks to an oscilloscopic display. An on-line LINC computer produced the stimulus display and recorded responses. The method was developed for the study of visual search, but it is applicable to other perceptual or cognitive problems.
\end{abstract}

B. F. Skinner wrote an interesting page in the technology of World War II by training his "pigeons in a pelican" to guide a missile (Skinner, 1960). The birds pecked at a hinged screen on which the missile's target was displayed. This ingenious invention illustrated the possibilities inherent in a technique for sensing the location of pecks to a complex image, but methods for presenting stimulus materials and recording peck locations have remained relatively crude and inflexible (e.g., Jenkins \& Sainsbury, 1970). The method described here incorporates a small computer and other standard apparatus in a relatively efficient and flexible system. A photocell mounted on a pigeon's beak functions as a "light-pen" to indicate the time and location of the bird's pecks at an oscilloscopic display. As reported here, the technique has been used to study visual search, but it appears to be adaptable to other tasks related to perception and information processing.

The usefulness of additional methods for studying perception and information processing in animals seems beyond question, for many interesting problems in these areas are not uniquely tied to human subjects. The pigeon seems a particularly appropriate subject

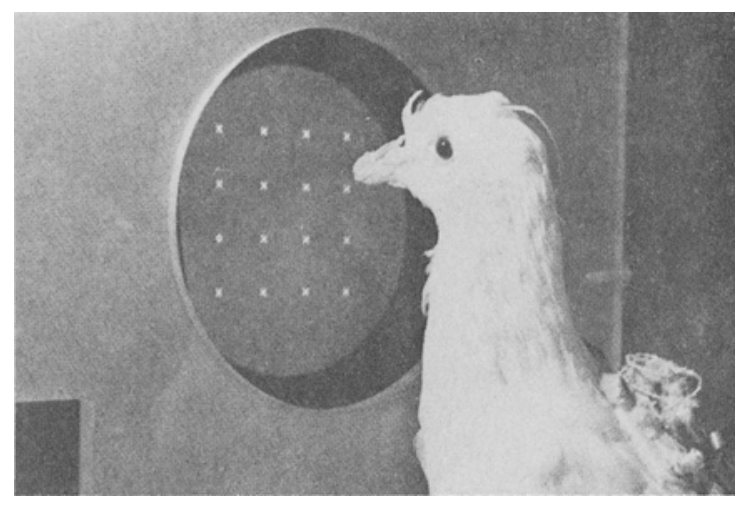

Figure 1. A pigeon confronts one of the displays used in a search experiment. The photocell makes a slight lump on the beak; lead wires are visible behind the head. During experimentation, illumination comes only from the display. with which to investigate the generality of visual information processing mechanisms. Its visual system is quite different from the human, yet is highly developed. The pigeon has good acuity, and something is known of its other visual functions. It is, of course, familiar as a hardy and convenient behavioral subject.

For the sake of concreteness, the technique is described here as it was employed in studies of visual search. Other applications would probably require changes mainly in the stimulus display. For the search experiments, an on-line computer generated a pattern of forms upon the screen of a cathode-ray oscilloscope. In a typical display, a single small " 0 " might appear among a number of small " $x$ " forms (Figure 1). To receive food reinforcement, the pigeon had to locate and peck the "o." The time from display onset until the peck, and the number of errors (pecks at " $x$ "s) were the dependent variables.

\section{CENTRAL CONTROL}

We have used the LINC computer (Clark \& Molnar, 1964) to produce the stimulus display, record responses, and analyze data. Though 12 years old, this is a versatile and efficient machine. Its memory is very small $(2 \mathrm{~K})$, but less than 1,000 program locations are used to conduct the experiments. Modern small computers are faster and have much larger memories; if equipped with analog output converters they should be readily adaptable to this use. We have also used the LINC analog input, but this capability is not essential, for responses could be sensed with a properly adjusted trigger circuit.

The LINC identified responses by noting the correspondence in time between the execution of a display instruction and the detection of an input pulse from the beak-mounted photocell. In the typical search display program, two LINC "display character" (DSC) instructions caused a single stimulus form to appear. These instructions were followed immediately by a "sample" (SAM) instruction that put an input voltage from the photocell into the machine's accumulator. If the input voltage was above a preset level, a criterion input was recorded. Such an event would normally 
mean that the pigeon's beak was near the character just displayed. Further details of this process are outlined below.

\section{DISPLAY}

A Tektronix Type 503 oscilloscope carried the display; cathode-ray tubes with P2 and P31 phosphors have been used successfully. The latter, a standard fast phosphor, has less troublesome afterglow; even so, faint replicas of displayed forms could remain visible if not overwritten by a blanking pattern. The LINC generated a flicker-free 16-character matrix on the display scope, refreshed approximately 154 times per second; more modern machines would improve this speed. Two LINC "DSC" instructions produced each display character by selectively intensifying points within a square 4 by 4 matrix. The geometry of the display was controlled by additional LINC instructions. The overall size was adjusted by amplification settings at the oscilloscope, as was the intensity. The search experiments used characters 4-mm in diam. Character luminance was about $2 \mathrm{~cd} / \mathrm{m}^{2}$, as measured with an SEI photometer viewing a solid square character composed of 16 dots. The temporal characteristics of individual display flashes were not determined independently of the input photocell and amplification system. That system and the nature of the input signal are described below.

Because the pigeon's peck is so rapid, one might wonder if the input system could miss responses. Highspeed photos of pigeon pecks by Hodos (Note 1) show that, under his experimental conditions (responding on a ratio schedule), the bird's beak was against the target for roughly $15 \mathrm{msec}$, and within a few millimeters of the target for perhaps twice that period. At 154 stimulus flashes per second, this leaves the beak within range for about five flashes. Our records of input during pecking confirmed that each peck produced several usable input pulses. Thus, it is unlikely that responses are missed because the stimulus fails to flash while the beak is near the screen. Instead, the factor limiting the number of elements or the complexity of the pattern displayed is probably the appearance of flicker. R.W. Powell (reported in Smith, 1970) found pigeon critical flicker frequencies as high as $140 \mathrm{~Hz}$, with very high intensities and small pulse-to-cycle fraction. In the LINC display, the minimum time between stimulus flashes in a given location increases almost linearly with the number of elements displayed; so, depending on intensity, a rather low number of elements can be presented free of visible flicker. Modern fast display systems should permit the display of more material.

The display appeared to the bird through a hole in an experimental chamber, as shown in Figure 1. The screen was set back $3 \mathrm{~cm}$ behind the wall of the chamber, for some birds tend to press against the display and obscure it with their feathers. Other features of the chamber are not critical. The food magazine was located below and to the left of the display. A central location would be preferable but is difficult because the oscilloscope is in the way. The chamber was constructed from clear plastic, so that the pigeon could be observed easily from various angles. Since the display was silent, masking noise was used only to prevent disturbance from room noises.

\section{INPUT}

As shown in Figure 2, the input photocell was mounted on the pigeon's upper mandible. The photocell (Amperex OAP-12, 9-mm long, 3-mm diam) was held in place with a small amount of dental acrylic, including a small bridge of the cement across the lower side of the mandible. The unit, including mounting cement, weighed approximately $.5 \mathrm{~g}$, and it appeared to interfere with neither eating nor head movement. The mounting tended to come loose after a time. When this occurred, it was reattached with a bit of acrylic or bonded to the beak with Krazy Glue, one of several instant-bonding brands that adhere well to the skin.

The mobility of the pigeon's head creates something of an input transmission problem. The best arrangement so far devised is the following. With the bird under light nembutal anesthesia (about $25 \mathrm{mg} / \mathrm{kg}$ intravenous, injected into the vein prominent under the bird's wing), 1-mm polyethylene tubing was pulled under the scalp, leaving one end protruding just above the beak and the other at the back of the head. The tubing was flared at each end with a hot nail, and dabs of acrylic cement at the ends prevented the tubing from pulling out. Two fine $(.28-\mathrm{mm})$ nylon-insulated stainless steel stranded wires (Bergen 302) were threaded through this tubing. These were attached to the photocell leads with stainless steel solder. At the other end, several

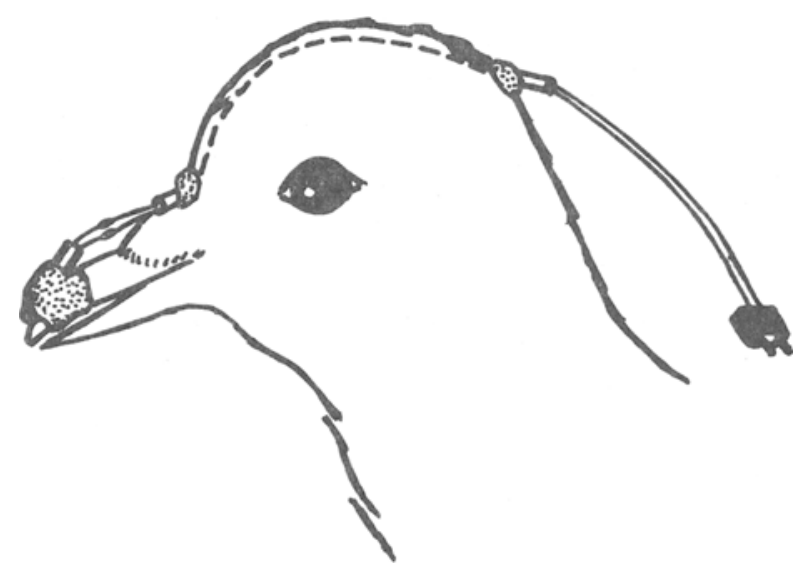

Figure 2. Photocell is mounted on the upper mandible, with some acrylic bridging below the mandible, making a ring of cement. A somewhat less successful altemative mounting dispensed with the subcutaneous tube; the plug was mounted on the pigeon's head with a "cap" of cement. 
centimeters beyond the back of the head, the wires were soldered to a small two-prong plug. A mating plug connected through flexible wire to a larger plug mounted on a small backpack. The pack mounting is not critical. We glued the plug to a scrap of plastic and harnassed this to the bird with a soft heavy string under each wing. From a plug mating with the backpack connector, heavy shielded wire led through a hole in the top of the experimental chamber to the amplifier. Our birds turned very little in the chamber and the input lead did not become twisted. If necessary, a mercury swivel can be used to overcome twisting.

The wire from the bird's beak must be light weight, strong, and flexible; stranded stainless steel wire appears to be ideal. The wire must be disconnected from the backpack when the bird is not being run, for otherwise the bird gets the wire twisted around its neck or caught in its beak.

Pigeons reinforced with food peck at the displayed characters with a biting motion (cf. Hearst \& Jenkins, 1974), so that the upper mandible strikes above the target form. Because of this, the input photocell was mounted in a slanting orientation, as shown in Figure 3. The glass lens of the OAP- 12 photocell provided highly directional sensitivity to signals from the display. Figure 3 suggests, for a typical sensitivity setting of the input system, the approximate area within which a display causes a response to be recorded. As the

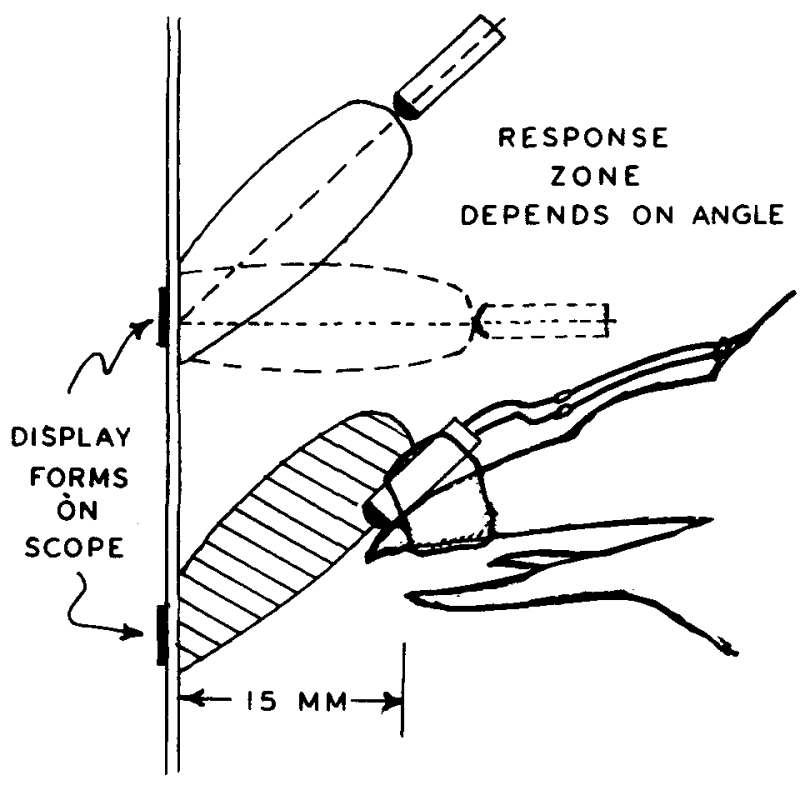

Figure 3. Diagram showing typical response characteristics of the photocell. The oscilloscope screen is represented at left, with the location of two 4-mm display forms shown to scale. The oval areas suggest the approximate region within which a photocell, oriented at the indicated angle, triggers a response. This area shrinks when the display intensity or input amplification decreases, and expands when intensity or amplification increases. figure suggests, the directional response of the photocell and its mounting angle combined to compensate for the fact that the upper mandible usually approached the screen above the target. Figure 3 also shows, to scale, the size and typical separation of targets used in the visual search task.

The Amperex OAP-12 is a germanium photodiode designed for sound-film scanning and control purposes. We used the simplest possible input connection, which employs the voltage generated by the photocell when exposed to light. The two leads from the photocell were led directly to the input of the first of two ac amplifiers connected in series (Tektronix FM-122), set to a combined nominal gain of 100,000. This hook-up required a relatively bright display and high-gain amplification, but it had low noise and was quite satisfactory. Preliminary tests indicate that, connected as a photoconductor (in series with a battery and resistor of about 2 megohms), the OAP-12 photocell provides a strong signal adequate for work with dim displays. We have not determined the optimal circuit for this application.

The OAP-12 has a nominal frequency response of $50,000 \mathrm{~Hz}$, fast enough to be sensitive to the flashes of individual points in the displayed forms. In our application, the input system slowed this response and integrated the effect of points within a single form, so that different forms did not produce markedly different inputs, and so that the input pulse lasted long enough to be sampled by the computer. This timing adjustment was done with the high-pass controls on the Tektronix amplifiers, which were set to $1,000 \mathrm{~Hz}$ (high); low pass was set to its highest value $(80 \mathrm{~Hz})$ to suppress 60 -cycle hum. The signal from a display form peaked in approximately $.2 \mathrm{msec}$ and was above baseline for approximately $.35 \mathrm{msec}$. We have used forms with the same number of intensified points so that they would be equally luminous to the subjects. Forms with substantially fewer points produce a lower input signal that might be troublesome in some applications.

The output from the second amplifier reached the LINC analog input through a $.001 \mathrm{mfd}$ capacitor; the signal at this point was about $1 \mathrm{~V}$. The LINC "SAM" instruction placed a digital transformation of this signal in the computer's accumulator. Sensitivity adjustment was possible at this point, since the computer was programmed to record a response only for inputs above a preset level. As an additional measure of noise rejection, the LINC was programmed to record a response only if it received two criterion inputs in succession, each correlated in time with the same display element.

\section{SUBJECT TRAINING}

Pigeons learn rapidly to peck at signals for food, and they are very persistent in this behavior (Hearst 
\& Jenkins, 1974). In accordance with the familiar "autoshaping" paradigm, all that is required to initiate pecking is the repeated appearance of a target form, followed within a few seconds by food presentation. A long interpresentation interval (more than $1 \mathrm{~min}$ ) facilitates the initiation of pecking. Forms not followed by food are not pecked; discrimination between the 4-mm " $x$ " and " $o$ " forms in our search experiments began to appear within a single session. Once the discrimination was learned, few errors were made and performance was easily maintained by intermittent reinforcement (probability 1/12).

\section{EVALUATION OF THE METHOD}

If one has access to an on-line computer, the method described here is not difficult to implement and has considerable merit. It provides a reasonably adequate solution to the problem of response localization. Equally important, it uses the great convenience and flexibility of the computer-driven display. The experimenter controls the form, location, time course, and movement of stimuli by programming, rather than with complex mechanical and optical equipment. The technique is applicable to an indefinite variety of problems that can be formulated as the detection or choice of visual stimuli.

Certain limitations of the technique should be noted. Displays are restricted to those that can be produced by intensifying points on an oscilloscope screen, within the time constraints imposed by computer speed and flicker. This eliminates colored displays and complex pictures, for example. (However, it might prove feasible to superimpose on a computer-generated display other material not under direct computer control.) Because the photocell signal varies with stimulus intensity, it is awkward to use intensity as an independent stimulus variable, and, in some applications, the need to equalize photocell input from different display forms could be troublesome. Because some birds tend to peck inaccurately (pecking too high is common), users may experience difficulty with problems that call for great response accuracy, as might be required with closely spaced target forms. Finally, because available display and input equipment vary, the potential user should expect to spend some time discovering the optimal adjustments. If a reasonably extended experiment is in prospect, this effort should be amply repaid.

\section{REFERENCE NOTE}

1. Hodos, W. Personal communication, 1976.

\section{REFERENCES}

Clark, W. A., \& Molnar, C. E. The LiNC: A description of the laboratory instrument computer. Annals of the New York Academy of Sciences, 1964, 115, 653-668.

Hearst, E., \& Jenkins, E. H. Sign tracking: The stimulusreinforcer relation and directed action. Austin, Tex: The Psychonomic Society, 1974.

Jenkins, H. M., \& SAinsbury, R. S. Discrimination learning with the distinctive feature on positive or negative trials. In D. Mostofsky (Ed.), Attention: Contemporary theory and analysis. New York: Appleton-Century-Crofts, 1970.

Sxinner, B. F. Pigeons in a pelican. American Psychologist, $1960,15,28-37$.

Smith, J. Conditioned suppression as an animal psychophysical technique. In W. C. Stebbins (Ed.), Animal Psychophysics. New York: Appleton-Century-Crofts, 1970.

(Received for publication October 15, 1976; revision accepted December 20,1976 .) 